\title{
VOLTAMETRIA DE MICROPARTÍCULAS IMOBILIZADAS: FUNDAMENTOS E APLICAÇÕES ANALÍTICAS
}

Leandro M. de Carvalho*, Maurício Hilgemann, Cristiane Spengler, Paulo C. do Nascimento e Denise Bohrer

Departamento de Química, Universidade Federal de Santa Maria, CP 5051, 97110-970 Santa Maria - RS, Brasil

Recebido em 9/11/09; aceito em 17/4/10; publicado na web em 1/9/10

\begin{abstract}
VOLTAMMETRY OF IMMOBILIZED MICROPARTICLES: FUNDAMENTALS AND ANALYTICAL APPLICATIONS. The theoretical aspects of Voltammetry of Immobilized Microparticles (VIM) were discussed. The immobilization of microparticles on electrode surface, the electrode cleaning processes and the electrode materials were analyzed. The three-phase electrode model and the possible reactions between the immobilized particle and the electrolytic solution were discussed. In addition, this work discusses some selected applications of VIM published in the last years.
\end{abstract}

Keywords: voltammetry of immobilized microparticles; solid materials; three-phase electrode.

\section{INTRODUÇÃO}

Tradicionalmente, a eletroanalítica está associada aos estudos de substâncias dissolvidas em fases líquidas. Desde a descrição da voltametria por Kolthoff e Laitinen, em 1940, ${ }^{1}$ este método analítico tornou-se o mais importante no campo da eletroquímica. Entretanto, para que estudos eletroanalíticos de substâncias sólidas fossem possíveis, novas estratégias experimentais e conceitos teóricos tiveram que ser aprimorados a fim de superar os problemas associados com a preparação da amostra e a eletroquímica de fases sólidas.

Em 1989, as dificuldades experimentais puderam ser superadas com o surgimento de uma nova técnica desenvolvida por Scholz e colaboradores, ${ }^{2,3}$ chamada inicialmente de voltametria abrasiva de redissolução (AbrSV - Abrasive Stripping Voltammetry) e, posteriormente, de voltametria de micropartículas imobilizadas (VIM - Voltammetry of Immobilized Microparticles $).{ }^{4}$ Esta técnica eletroanalítica baseia-se na imobilização de micropartículas de uma amostra sólida (menos de $1 \mu \mathrm{g}$ ) sobre a superfície de um eletrodo sólido adequado, o qual é imerso em um eletrólito líquido, e no subsequente registro de um sinal de corrente em função do potencial aplicado ${ }^{5,6}$ Os voltamogramas obtidos revelam informações sobre a composição química da amostra sólida.

A imobilização de micropartículas tem se tornado um método bastante utilizado para ensaios diretos de sólidos devido à facilidade de preparação do eletrodo, evitando a dissolução da amostra, além de possibilitar uma análise rápida e de baixo custo. ${ }^{7}$ Além de ser um método indicado para determinações qualitativas e semiquantitativas, ${ }^{8,9}$ também fornece informações sobre a cinética das reações redox de materiais sólidos. ${ }^{4,10}$

Encontram-se, na literatura, textos dedicados exclusivamente à VIM, a exemplo de capítulos em livros-texto, ${ }^{4,11}$ de alguns artigos de revisão publicados em periódicos especializados, ${ }^{7,9,12}$ e um livro-texto dedicado aos seus fundamentos e aplicações, publicado por Scholz, Schröder e Gulaboski intitulado "Electrochemistry of Immobilized Particles and Droplets". ${ }^{13}$

Este artigo apresenta uma revisão sobre os fundamentos que envolvem a determinação voltamétrica de materiais sólidos e suas principais aplicações analíticas. Os principais tipos de eletrodos utilizados são descritos, assim como a sua limpeza e a imobilização mecânica de micropartículas à sua superfície. O trabalho apresenta uma discussão sobre os avanços conseguidos pela VIM para a aplicabilidade da eletroquímica no estudo dos processos redox envolvendo amostras sólidas.

*e-mail: lemacarvalho@gmail.com

\section{FUNDAMENTOS DA VIM E MEDIDAS VOLTAMÉTRICAS}

A eletroquímica fornece-nos um poderoso arsenal de técnicas que podem ser usadas no estudo de soluções líquidas de eletrólitos, eletrólitos sólidos, eletrodos metálicos e semicondutores, assim como condutores de íons e elétrons. A questão mais importante na decisão sobre a escolha de uma determinada técnica eletroquímica sempre foi como tornar possível o estudo de um eletrodo ou de uma solução de um composto. Esta exigência trouxe severas limitações para uma aplicabilidade geral da eletroquímica. Ao mostrar que a imobilização mecânica de partículas sólidas na superfície de eletrodos inertes permite a realização de praticamente todas as medidas eletroquímicas de determinado composto ou material, desde que ele não se dissolva na solução do eletrólito, foi dado um passo marcante na aplicabilidade da eletroquímica. ${ }^{13}$ Neste contexto, a VIM ampliou as perspectivas em relação aos estudos de amostras sólidas devido às suas variadas possibilidades de aplicação. Em princípio, qualquer amostra sólida e insolúvel contendo elementos que possam sofrer reações eletroquímicas é adequada para ser analisada por VIM. ${ }^{4}$ A aplicação da VIM baseia-se no fato de que, pela Lei de Faraday, quantidades extremamente pequenas de uma amostra são suficientes para gerar, facilmente, correntes mensuráveis. ${ }^{12}$

A aplicação eletroquímica apresentada nesta revisão é bastante diversa; entretanto, há uma característica comum: todos os eletrodos com partículas eletroativas imobilizadas apresentam as transferências eletrônica e iônica ocorrendo simultaneamente. Tais eletrodos são chamados de "eletrodos de três fases", uma vez que as três fases estão em contato. Este modelo também se aplica para a maioria dos eletrodos de filme ou eletrodos com superfície modificada, muitos eletrodos de baterias e células a combustível, eletrodos de segunda classe, etc. De fato, a maior parte dos eletrodos com superfície modificada possui um arranjo de partículas que cobrem parcialmente o eletrodo. ${ }^{13}$

A Figura 1 mostra a representação do eletrodo de três fases, em que a fase II é uma partícula. Uma vez que a partícula contém moléculas neutras e íons com quantidades idênticas de cargas positivas e negativas, qualquer transferência de elétrons entre as fases I e II deve ser acompanhada por uma transferência de íons entre as fases II e III. A transferência de íons é uma reação indispensável para manter a eletroneutralidade da fase II. A transferência de elétrons entre as fases I e II pode ser ignorada apenas no caso de uma oxidação metálica simples. Um exemplo de uma reação eletroquímica em um sistema de 3 fases consiste de grafite (fase I), azul da Prússia (fase II) e a solução do eletrólito (fase III). Elétrons serão trocados entre o grafite (fase 
I) e o azul da Prússia (fase II), enquanto cátions $\mathrm{K}^{+}$serão trocados entre o azul da Prússia (fase II) e a solução do eletrólito (fase III). ${ }^{14}$

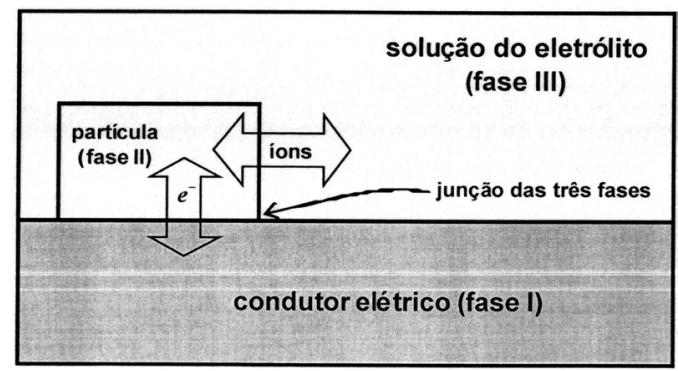

Figura 1. Desenho esquemático do sistema eletródico de três fases: interface eletrodo/composto/solução, no qual o eletrodo de trabalho contém uma partícula aderida à sua superfície. As setas horizontal e vertical representam as transferências iônica e eletrônica entre as fases ocorrendo simultaneamente

\section{Eletrodos de trabalho}

Na VIM, geralmente todos os tipos de materiais podem ser usados como eletrodos sólidos. ${ }^{12}$ No entanto, a escolha do eletrodo depende de sua natureza, que está relacionada à habilidade do sólido de aderir à superfície do eletrodo.

$\mathrm{O}$ grafite tornou-se o material base mais utilizado como eletrodo na VIM. ${ }^{4,13}$ Ele combina uma grande inércia química, com uma ampla janela de potenciais, baixo preço e facilidade de processamento. Uma das grandes vantagens dos eletrodos à base de grafite é a facilidade de limpeza do eletrodo através da remoção da superfície inferior do eletrodo por abrasão ou corte, quando amostras pulverizadas estão imobilizadas a ele.

O eletrodo de grafite impregnado com parafina (PIGE) mostrou-se bastante adequado para fixar quantidades pequenas de partículas sólidas na superfície do eletrodo. Ele é feito de grafite de alta pureza, cujos poros são preenchidos com parafina para prevenir altas correntes de fundo e a contaminação do eletrodo de trabalho através da penetração da solução do eletrólito. O tamanho típico do eletrodo de grafite utilizado em experimentos de VIM é de $5 \mathrm{~cm}$ de comprimento e $5 \mathrm{~mm}$ de diâmetro. ${ }^{4}$

A introdução de eletrodos de pasta de carbono (CPE) foi uma importante etapa na eletroanálise em estado sólido. Tradicionalmente, CPEs são constituídos de carbono com um aglutinante orgânico (Nujol ou óleo de silicone). Se o sólido a ser estudado é adicionado à pasta, o eletrodo é chamado "CPE modificado" ou "eletrodo eletroativo de pasta de carbono" (CPEE). ${ }^{7}$ A técnica de eletrodos de pasta modificados foi extensamente desenvolvida na ex-União Soviética por Brainina. ${ }^{15,16}$ A metodologia de CPEE aumentou significativamente o escopo da eletroquímica de sólidos condutores e insolúveis e possibilitou as primeiras aplicações da eletroquímica no estado sólido. Atualmente, CPEEs são principalmente empregados nos estudos de interações de sólidos com espécies dissolvidas.?

Eletrodos compósitos à base de pó de grafite como material base usualmente requerem aglutinantes à base de resina polimérica, como poliéster, ${ }^{17,18}$ ou cera, ${ }^{19}$ a fim de selar os poros e conferir estabilidade mecânica ao eletrodo. A escolha do aglutinante apropriado é crucial, pois a inércia química e física contra a solução do eletrólito, a estabilidade térmica e as propriedades mecânicas são predominantemente regidas pelo aglutinante. O processo de preparação do eletrodo compósito é geralmente simples. Consiste na mistura de pó de grafite e do aglutinante, sendo que a razão entre as massas dependerá do aglutinante usado, da estabilidade mecânica desejada para o eletrodo e do processo de solidificação. Este último pode ser acompanhado da cura da resina polimérica, ${ }^{18}$ ou pela ressolidificação da cera ou parafina fundidas.

O eletrodo de carbono vítreo (GCE) é menos indicado para a imobilização mecânica, pois partículas não aderem facilmente à sua superfície. Exceções são compostos orgânicos e organo-metálicos que conseguem fixar-se à superfície do carbono vítreo. ${ }^{20} \mathrm{Em}$ um trabalho de Lange e colaboradores, ${ }^{21}$ foi demonstrado que a aderência de micropartículas ao GCE é muito mais fraca que em eletrodos de grafite, em que as partículas são literalmente incorporadas à superfície do eletrodo. Entretanto, os eletrodos de carbono vítreo têm sido deliberadamente escolhidos para a imobilização de partículas a fim de se ter uma camada de eletrólito prensada entre o carbono e as partículas, para se estudar a catálise da transferência de elétrons de íons e compostos adsorvidos à superfície da partícula. ${ }^{21}$

Eletrodos de carbono pirolítico ${ }^{22,23}$ são muito adequados para a voltametria de micropartículas. Como a solução do eletrólito não penetra o carbono pirolítico, este não requer nenhum aglutinante orgânico como selante, o que o faz igualmente adequado para medidas em meios aquoso e orgânico. ${ }^{13} \mathrm{O}$ eletrodo de carbono pirolítico altamente orientado (HOPG) possui em sua estrutura uma camada muito frágil, que proíbe a imobilização mecânica de partículas que poderiam danificar severamente a superfície do material. Contudo, o HOPG é o material preferencial para análises de microscopia de varredura eletroquímica (SECM) e microscopia de força atômica (AFM), e tem sido usado com sucesso na investigação microscópica de micropartículas sólidas. ${ }^{13,24}$

Atualmente, eletrodos de diamante dopados com boro ${ }^{25,26}$ têm recebido muita atenção na VIM, pois sua extrema dureza permite a amostragem direta de materiais duros como metais e cerâmica. Propriedades desejáveis como alta inércia química associada à ampla faixa útil de potenciais, à baixa capacidade interfacial em eletrólitos aquosos e, portanto, baixas correntes de fundo, agregam valor especial a estes eletrodos. Todavia, a limpeza destes eletrodos após a imobilização mecânica (abrasiva) da amostra é bastante tediosa. ${ }^{13}$

O eletrodo compacto (CE, Compact Electrode) é um corpo compacto, como uma pastilha, disco, ou haste, feito do material a ser estudado, usado como eletrodo de trabalho. ${ }^{7}$ Eletrodos compactos são apenas indicados para bons condutores elétricos, como metais, $\mathrm{Fe}_{3} \mathrm{O}_{4}{ }^{27,28}$ ou $\mathrm{UO}_{2} \cdot{ }^{29,30}$ Esta técnica também foi estudada para calcogenetos como $\mathrm{Cu}_{\mathrm{x}} \mathrm{S},{ }^{31} \mathrm{PbS}^{32}$ e FeAsS. ${ }^{33} \mathrm{~A}$ necessidade de estudar materiais pouco condutores revelou o limite dos eletrodos compactos: a resistência ôhmica entre a superfície ativa do sólido e o coletor de corrente causa distorção nas curvas voltamétricas. ${ }^{13}$ Aplicações analíticas de eletrodos compactos são um tanto excepcionais, pois sua preparação é geralmente laboriosa e requer procedimentos especiais, como compressão e sinterização. Sob alta pressão e temperatura, a estequiometria e a composição de fases das amostras pulverizadas a serem analisadas podem alterar. A técnica com eletrodos compactos é, hoje em dia, principalmente usada para estudar a eletroquímica superficial de minerais que ocorrem naturalmente e sua interação com soluções.

Em virtude de sua dureza ser similar à do carbono vítreo, eletrodos metálicos são pouco adequados para a voltametria de micropartículas imobilizadas mecanicamente. ${ }^{13}$ Entretanto, eletrodos metálicos são usados com sucesso na investigação de sólidos orgânicos e organometálicos. Como foi demonstrado por Jiang e Kucernak, ${ }^{20}$ com eletrodos de ouro, carbono vítreo e platina, resultados muito semelhantes são obtidos na análise de microcristais de fito-halocianino de platina (PtPc).

\section{Imobilização de micropartículas}

O método de preparação da amostra sólida para uma medida eletroquímica não é uma tarefa padrão. Para a medida eletroquímica, as partículas da amostra devem ter um contato elétrico suficientemente bom com o eletrodo e também com a fase da solução fornecedora de íons. Por propósitos analíticos práticos, a técnica deve ser simples, confiável ou, pelo menos, adequada para uma faixa bem definida de aplicações.

Para a imobilização das micropartículas da amostra sólida na superfície do PIGE, a amostra é moída em um pó fino dentro de um gral com 
um pistilo. Uma pequena quantidade da amostra pulverizada é colocada em cima de um azulejo de porcelana vítreo, e a superfície inferior do eletrodo é pressionada com movimentos circulares durante alguns segundos sobre a amostra. Usualmente, isto resultará na fixação de uma gama de partículas ao eletrodo. A maior parte delas ficará parcialmente embutida na superfície do grafite. Outra parte ficará fixada por adesão; entretanto, uma parte pode aderir tão fracamente que poderá ser lixiviada com água. Como não restará água no eletrodo hidrofóbico, esta lavagem não irá diluir a solução do eletrólito. De fato, a lavagem de partículas que não aderem firmemente à superfície é desnecessária, pois elas irão cair do eletrodo após sua introdução na solução do eletrólito. ${ }^{13}$

Uma segunda maneira de imobilizar as partículas é colocar poucos miligramas da amostra pulverizada sobre papel filtro e friccionar o eletrodo sobre a amostra. Este procedimento tem como vantagem alcançar uma maior distribuição e, as partículas perdidas, ao mesmo tempo, são mantidas sobre o papel. Alguns experimentos preliminares darão a experiência necessária sobre a quantidade de amostra que deve ser utilizada, qual a pressão correta ao friccionar o eletrodo sobre a amostra e qual o melhor movimento para a fricção. Ocasionalmente, pode ser adequado apenas pressionar o eletrodo sobre a amostra, sem fazer movimentos circulares com ele. Quando metais e ligas são estudados, basta friccionar levemente o eletrodo à superfície destes, que podem ser, mas não necessariamente, previamente limpos. Quando metais e ligas são muito duros, um pó abrasivo (por exemplo, $\mathrm{Al}_{2} \mathrm{O}_{3}$ de alta pureza ou diamante) pode ser colocado entre o eletrodo e a amostra compacta. Devido à sua inércia, este tipo de pó abrasivo não interferirá na eletroquímica. Quando a amostra é um pó bastante grosseiro, o melhor é triturar uma pequena quantidade em um gral para se conseguir pequenas partículas. ${ }^{13}$

Esta imobilização mecânica da amostra sólida na superfície do eletrodo se assemelha à etapa de pré-concentração de análises voltamétricas de redissolução. No entanto, diferentemente da adsorção controlada realizada durante as análises voltamétricas de redissolução, a quantidade de amostra incorporada na VIM é desconhecida, variando entre 0,1 e 2 $\mu \mathrm{g}$, o que restringe a quantificação direta de espécies eletroativas.

\section{Limpeza do eletrodo de trabalho}

Após realizar-se a medida eletroquímica, a limpeza da superfície do eletrodo para a próxima medida é de fundamental importância. A melhor forma de limpar o PIGE é friccionar sua superfície sobre um papel filtro. Esta fricção deve ser interrompida sobre o papel, com isso a amostra remanescente não consegue ser transportada de uma ponta à outra. O eletrodo deve ser polido mantendo-o perpendicular à superfície do papel para evitar o arredondamento das laterais. A superfície do eletrodo deve ser mantida plana, pois isto ajuda bastante nas operações de limpeza subsequentes. Algumas vezes pode ser aconselhável dobrar um pedaço de papel filtro limpo ao redor da haste do eletrodo para retirar possíveis resquícios de amostra que possam ter sido transferidos a ele durante o polimento. Depois disso, sugere-se polir a superfície circular inferior novamente. Os resultados da limpeza devem ser conferidos obtendo-se um voltamograma do branco (eletrólito suporte), o qual não deve apresentar picos. Sem isto, não há como assegurar que a resposta da medida da amostra é causada apenas pela amostra em questão e não como resultado de medidas anteriores. Em alguns casos raros, quando as partículas são muito duras (isto acontece com algumas partículas de aço), partículas remanescentes podem ser transportadas adiante no eletrodo e não é possível se livrar delas por este procedimento de polimento. Neste caso deve-se remover uma fina camada do eletrodo em um papel abrasivo muito fino, seguido pelo polimento da superfície do eletrodo sobre o papel. A superfície do eletrodo também pode ser limpa com a ajuda de uma lâmina de barbear. O melhor movimento para polir a superfície do eletrodo é escrever o número " 8 " sobre o papel, mantendo, entretanto, o eletrodo o mais ereto possível. ${ }^{13}$

Devido à limpeza e polimento da superfície do eletrodo, o PIGE diminuirá gradualmente de tamanho depois de cada experimento. Entretanto, isto não tem nenhum efeito significativo sobre a medida, sendo que cada eletrodo pode ser usado inúmeras vezes. O mesmo é válido para a solução de eletrólito, já que a quantidade da solução em relação ao sólido é muito grande, e um grande número de análises pode ser realizado antes que o eletrólito sature ou interfira nas análises. ${ }^{13}$

\section{Medida eletroquímica}

Após a imobilização da amostra sólida por abrasão na superfície de um eletrodo adequado, o eletrodo de trabalho é inserido na célula eletroquímica contendo os eletrodos de referência e auxiliar. $\mathrm{O}$ design da célula depende do eletrodo de trabalho utilizado e do propósito do experimento, podendo-se empregar desde células de vidro convencionais até construções especialmente adaptadas para experimentos em fluxo ou para espectroeletroquímica. ${ }^{13}$

A escolha do eletrólito suporte depende da natureza do composto e do processo estudados. Na maior parte dos casos, eletrólitos aquosos são usados na VIM. Entretanto, solventes orgânicos como acetonitrila também podem ser utilizados, uma vez que sua constante dielétrica permite que eletrólitos salinos sejam dissolvidos a fim de se obter a condutividade necessária na solução. O principal pré-requisito na escolha do solvente do eletrólito é a baixa solubilidade do composto a ser estudado no solvente. , $^{413}$

As reações eletroquímicas na VIM se processam na interface eletrodo/composto/solução, como ilustrado na Figura 1. Em contraste com outras aplicações voltamétricas, somente a superfície circular da ponta do eletrodo de trabalho (PIGE, por exemplo) entra em contato com a solução do eletrólito. Isto assegura que as reações de oxi-redução aconteçam somente na junção das três fases (eletrodo/ composto sólido/solução do eletrólito), além de garantir que a área superficial do eletrodo seja a mesma de experimento para experimento. Portanto, os compostos eletroativos presentes na amostra imobilizada trocam elétrons com o eletrodo de trabalho e íons com a solução do eletrólito, preservando, assim, o equilíbrio de cargas em cada fase. Além disso, o baixo contato da superfície do eletrodo com a solução do eletrólito minimiza a penetração do eletrólito no corpo do eletrodo, além de diminuir a linha base e aumentar a relação sinal/ruídNeste sistema de três fases, algumas reações possíveis das partículas imobilizadas em um eletrodo em contato com uma solução eletrolítica são descritas a seguir (Figura 2). ${ }^{13}$

O caso 1 da Figura 2 mostra a redução de um sal metálico insolúvel a metal, por exemplo, $\mathrm{AgCl}$ a $\mathrm{Ag}$, com os ânions liberados difundindo através do seio da solução do eletrólito. Para a redução de haletos de prata e também óxidos de chumbo II, poder-se-ia mostrar que o eduto sólido é diretamente transformado no produto sólido. $\mathrm{O}$ segundo caso é a oxidação de um sal metálico com a liberação de íons metálicos através da solução do eletrólito. Um exemplo disto seria a oxidação de sulfito de cobre I a sulfito de cobre II. O caso 3 apresenta a oxidação do metal e formação de um composto metálico insolúvel, ou seja, sal, óxido ou complexo. Esta reação pode proceder via solução supersaturada do composto MX, como no caso da oxidação da prata em AgBr e AgI. No caso 4, a simples dissolução oxidativa anódica de uma partícula metálica é considerada. O caso 5 diz respeito à eletroquímica de inserção de uma partícula em que íons são trocados entre a partícula sólida e a solução do eletrólito, quando elétrons são trocados entre a partícula e o eletrodo. O caso 6 mostra o caso em que a reação eletroquímica é confinada apenas à camada superficial. No caso 7, assume-se que as reações eletroquímicas ocorrem apenas via dissolução de uma partícula sólida já existente, 


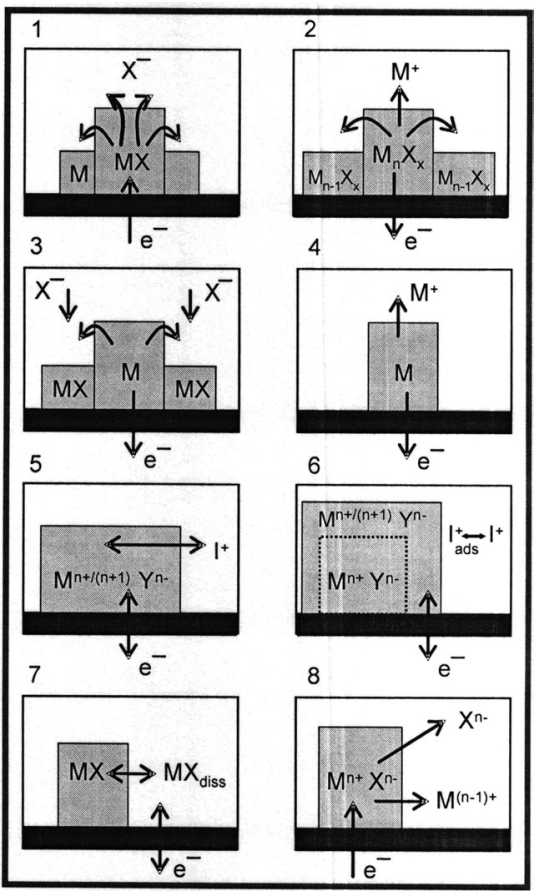

Figura 2. Esquema demonstrativo das possíveis reações eletroquímicas de partículas sólidas imobilizadas em um eletrodo sólido e em contato com a solução eletrolítica: (1) redução de um sal metálico insolúvel a metal; (2) oxidação de um sal metálico com a liberação de íons metálicos; (3) oxidação do metal e formação de um composto metálico insolúvel; (4) dissolução oxidativa anódica de uma partícula metálica; (5) eletroquímica de inserção de uma partícula; (6) reação eletroquímica confinada à camada superficial; (7) reação eletroquímica via dissolução de uma partícula sólida já existente e, (8) dissolução eletroquímica completa de uma partícula resultante de sua oxidação ou redução. Adaptado da ref. 13

e o caso 8 simboliza a dissolução eletroquímica completa de uma partícula resultante de sua oxidação ou redução. Um exemplo deste último tipo é a dissolução redutiva de óxidos de ferro III.

Na VIM, um potenciostato é usado para variar o potencial do sistema a uma velocidade constante durante a etapa de redissolução, para produzir uma resposta de corrente no eletrodo de trabalho, sendo que todas as técnicas voltamétricas de medida (voltametria de varredura linear, de pulso diferencial, de onda quadrada e cíclica) podem ser aplicadas. A janela útil de potenciais é limitada pelas características químicas do eletrólito utilizado e pelo eletrodo de trabalho.

\section{APLICAÇÕES ANALÍTICAS}

Desde a primeira descrição deste método, uma variedade de compostos tem sido estudada, como ligas metálicas, ${ }^{2,34}$ minerais, ${ }^{6,7}$ diferentes óxidos, ${ }^{35,36}$ compostos orgânicos, ${ }^{37}$ pigmentos ${ }^{38,39} \mathrm{e}$ fármacos em pó. ${ }^{40-42}$ Com a VIM, informações sobre os compostos eletroativos presentes numa amostra sólida são obtidas pela varredura de potenciais e visualização dos picos de corrente, que indicam a ocorrência de reações eletroquímicas. O potencial de pico é característico do composto que reagiu, e a área do pico é proporcional à quantidade da espécie eletroativa. Desta forma, a identificação das espécies eletroativas nas amostras é feita pela comparação dos seus potenciais de pico com os de amostras sintéticas de composição conhecida. ${ }^{8}$

Existem apenas duas exigências para os compostos e materiais sólidos serem utilizados na VIM: ser insolúveis na solução do eletrólito e eletroativos. O mais importante é que não existe restrição a respeito da condutividade eletrônica. Até isolantes, como o fósforo branco, podem ser estudados, pois a reação eletroquímica que acontece na interface das três fases composto/eletrodo/solução pode gerar carga suficiente para produzir correntes mensuráveis. ${ }^{43}$ Podem-se distinguir três diferentes tipos de compostos: aqueles que não são eletroativos, aqueles que são destruídos irreversivelmente nas reações eletroquímicas, e aqueles que podem ser reversivelmente reduzidos e oxidados. Estes últimos compostos possuem a habilidade de trocar elétrons com o eletrólito e íons com a solução. ${ }^{4}$

\section{Aplicações da VIM na análise e caracterização de compostos inorgânicos}

\section{Análise elementar}

Uma vez que técnicas voltamétricas conseguem detectar correntes extremamente baixas causadas pela oxidação e redução de quantidades muito baixas de substâncias, era claro se supor que a transferência mecânica de um sólido à superfície de um eletrodo seria capaz de depositar uma quantidade suficiente de metal, liga ou qualquer outro material sólido à superfície do eletrodo para se realizar medidas eletroquímicas com quantidades traço. A primeira publicação baseada nesta ideia reportou a análise de ligas de chumbo-antimônio. ${ }^{2}$ A transferência foi realizada através da fricção da superfície inferior circular do PIGE sobre a superfície das ligas. Uma vez que a quantidade absoluta dos traços de liga transferidos por fricção não pode ser controlada e varia em alguma extensão de experimento para experimento, a avaliação dos voltamogramas foi baseada na porcentagem de corrente de cada pico em relação à soma das correntes de pico. A grande vantagem em realizar medidas eletroquímicas com quantidades traço de ligas é a alta resolução que pode ser alcançada com altas velocidades de varredura, pois os constituintes da liga conseguem ser completamente oxidados e dissolvidos um após o outro sem a sobreposição de sinais. ${ }^{13}$

Análises quantitativas foram, até agora, reportadas apenas para ligas binárias. Se houver mais de dois constituintes em uma liga, complicações maiores podem surgir, como compostos intermetálicos e formação de fases. Ligas, calcogenetos e outros compostos contendo elementos eletroquimicamente ativos, tais como $\mathrm{Ag}, \mathrm{Cu}$ ou $\mathrm{Pb}$, ou compostos, como óxidos de $\mathrm{Mn}$, Fe ou $\mathrm{Cr}$, têm sido frequentemente estudados pela VIM. ${ }^{7} \mathrm{Na}$ Tabela 1 estão listadas algumas aplicações analíticas de estudos eletroquímicos de sólidos e a Figura 3 ilustra um exemplo de análise elementar utilizando a VIM.

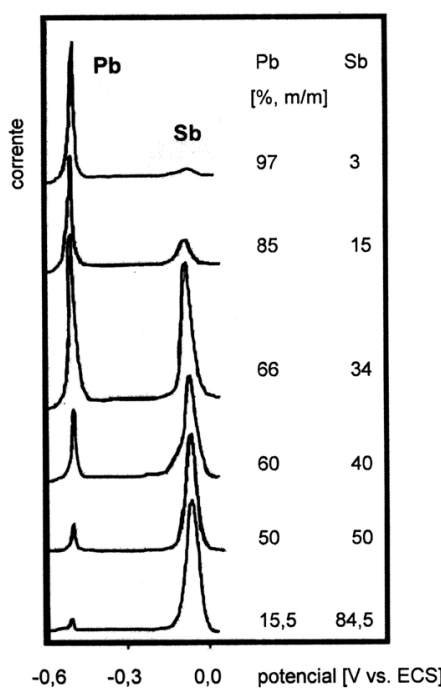

Figura 3. Voltamogramas de pulso diferencial obtidos após a transferência abrasiva de quantidades traço de diferentes ligas de Pb-Sb sobre um PIGE. Eletrólito suporte: ácido oxálico 0,1 mol $\mathrm{L}^{-1}$. Adaptada da ref. 13 
Tabela 1. Trabalhos envolvendo análises eletroquímicas em sólidos inorgânicos empregando VIM

\begin{tabular}{|c|c|c|c|}
\hline Analito & Propósito analítico & $\begin{array}{l}\text { Eletrodo de } \\
\text { trabalho }\end{array}$ & Ref. \\
\hline \multirow[t]{7}{*}{ Metais e ligas } & $\begin{array}{l}\text { Comportamento eletroquímico de ligas } \\
\qquad \text { de } \mathrm{Ag}-\mathrm{Cu}\end{array}$ & PIGE & 34 \\
\hline & Determinação de $\mathrm{Cu}$ & CPEE & 44 \\
\hline & Identificação de ligas metálicas dentárias & PIGE & 45,46 \\
\hline & Reatividade de Fe em pó & PIGE & 47,48 \\
\hline & Análise elementar de ligas $\mathrm{Pb}-\mathrm{Sb}$ & PIGE & 2 \\
\hline & Análise elementar de ligas $\mathrm{Sn}-\mathrm{Bi}$ & PIGE & 49 \\
\hline & $\begin{array}{l}\text { Estudo sobre as condições do processo de } \\
\text { abrasão de } \mathrm{Sn} \text { e Ag }\end{array}$ & $\begin{array}{l}\text { Diamante } \\
\text { dopado com } \\
\text { boro }\end{array}$ & 25 \\
\hline $\begin{array}{l}\text { Pigmentos } \\
\text { inorgânicos }\end{array}$ & $\begin{array}{l}\text { Identificação de íons metálicos em mate- } \\
\text { riais arqueológicos }\end{array}$ & Compósito & 17,50 \\
\hline $\begin{array}{l}\text { (óxidos, } \\
\text { calcogenetos) }\end{array}$ & $\begin{array}{l}\text { Identificação de íons metálicos em obras } \\
\text { de arte }\end{array}$ & Compósito & 51 \\
\hline \multirow[t]{3}{*}{ Calcogênios } & Estequiometria de $\mathrm{Cu}_{2-X} \mathrm{Se}$ & CPEE & 52 \\
\hline & Estequiometria de sulfetos de $\mathrm{CuS}_{X} \mathrm{Se}_{1-X}$ & PIGE & 53 \\
\hline & Estequiometria de sulfetos de $\mathrm{Pb}-\mathrm{Sb}$ & PIGE & 3 \\
\hline \multirow[t]{2}{*}{ Óxidos de $\mathrm{Cu}$} & $\begin{array}{l}\text { Identificação e caracterização de super- } \\
\text { condutores }\end{array}$ & PIGE, CPE & $57-59$ \\
\hline & $\begin{array}{l}\text { Pigmentos e produtos da meteorização de } \\
\text { trabalhos de arte }\end{array}$ & Compósito & 60,61 \\
\hline \multirow[t]{4}{*}{ Óxidos de Fe } & Análise de $\alpha-\mathrm{FeOOH}$ & PIGE & 62 \\
\hline & Comportamento eletroquímico & PIGE & 10 \\
\hline & $\mathrm{Fe}_{2} \mathrm{O}_{3}$ e $\mathrm{FeOOH}$ em solos antigos & PIGE & 63 \\
\hline & $\begin{array}{c}\text { Pigmentos em pedaços de trabalhos de } \\
\text { arte }\end{array}$ & $\begin{array}{l}\text { CPEE, } \\
\text { Compósito }\end{array}$ & 18,64 \\
\hline \multirow[t]{3}{*}{ Óxidos de Mn } & Mn em sedimentos marinhos & PIGE & 65 \\
\hline & Caracterização de materiais para baterias & GCE & 66 \\
\hline & $\begin{array}{c}\text { Identificação de } \mathrm{MnO}_{X} \text { em materiais } \\
\text { arqueológicos }\end{array}$ & Compósito & 67 \\
\hline \multirow[t]{2}{*}{ Óxidos de $\mathrm{Pb}$} & Comparação de vários óxidos de $\mathrm{Pb}$ & CPEE & 68 \\
\hline & $\begin{array}{c}\text { Identificação de } \mathrm{Pb}^{2+} \text { e } \mathrm{Pb}^{4+} \text { em cerâmicas } \\
\text { medievais }\end{array}$ & $\begin{array}{c}\text { CPEE, } \\
\text { Compósito }\end{array}$ & 69 \\
\hline $\begin{array}{l}\text { Compostos } \\
\text { de Pt }\end{array}$ & $\begin{array}{l}\text { Comportamento eletroquímico de catali- } \\
\text { sadores automotivos }\end{array}$ & CPEE & 70 \\
\hline Ti em zeólitas & $\begin{array}{c}\text { Especiação da estrutura de } \mathrm{Ti}^{4+} \mathrm{em} \\
\text { zeólitas }\end{array}$ & CPEE, GCE & $71-73$ \\
\hline
\end{tabular}

Análise de soluções sólidas

Soluções sólidas (misturas de sólidos) são de grande importância em vários campos científicos e tecnológicos. As análises de soluções sólidas devem responder a duas questões básicas: a solução sólida consiste de uma fase única ou é uma mistura (mecânica) de 2 fases diferentes? e, no caso de uma solução sólida de fase única, qual é a sua composição? Estas questões podem ser respondidas com a ajuda de várias técnicas analíticas, como difração de raios- $\mathrm{X}$ ou espectroscopia de infravermelho. Entretanto, a maior vantagem das investigações eletroquímicas de micropartículas imobilizadas é que estas respostas podem der encontradas com quantidades extremamente pequenas de amostra, até mesmo com uma única micropartícula. Na prática, um exemplo da eletroquímica de soluções sólidas é a situação em que a mistura de compostos individuais A e B produz dois sinais voltamétricos, enquanto que a solução sólida de $\mathrm{AB}$ exibirá um único sinal. ${ }^{4}$

Por exemplo, sulfeto e seleneto de cobre (II) formam séries contínuas de soluções sólidas $\mathrm{Cu}(\mathrm{S}, \mathrm{Se}) .{ }^{53}$ Quando imobilizados em um eletrodo de grafite, os compostos puros e as soluções sólidas podem ser reduzidos a cobre metálico, com íons sulfeto e seleneto sendo liberados e, dependendo do $\mathrm{pH}$ da solução, possivelmente protonados. A Figura 4 mostra os voltamogramas das reduções das fases puras $\mathrm{CuS}$ e CuSe, da solução sólida $\mathrm{CuSe}_{0,4} \mathrm{~S}_{0,6}$ e da mistura mecânica de $\mathrm{CuSe}$ e CuS. O voltamograma da solução sólida $\mathrm{CuSe} \mathrm{S}_{0,4} \mathrm{~S}_{0,6}$ exibe um único pico, como esperado para uma solução sólida.

a

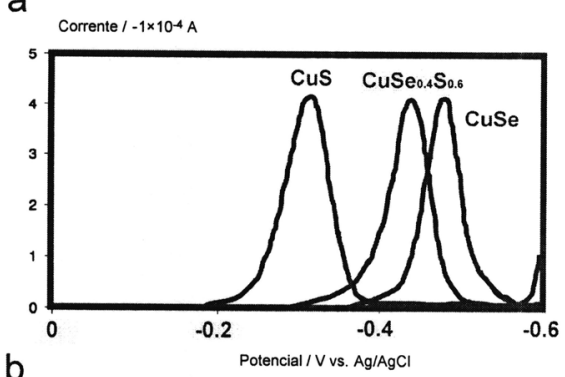

b

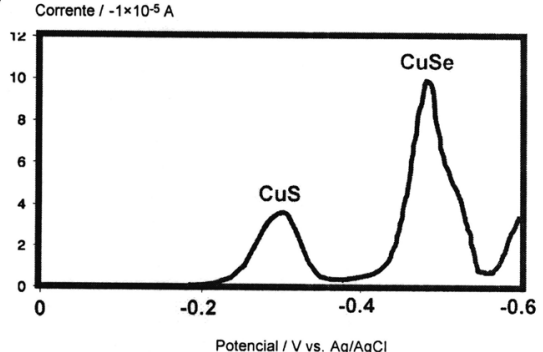

Figura 4. Curvas voltamétricas de (a) CuS, CuSe e CuSe ${ }_{0.4} S_{0,6}$ e (b) uma mistura mecânica de CuS e CuSe (1:2). Eletrólito suporte: $\mathrm{H}_{2} \mathrm{SO}_{4} 1 \mathrm{~mol} \mathrm{~L}^{-1}$; eletrodo de trabalho: PIGE. Adaptada da ref. 13

Análise da estequiometria de óxidos metálicos e calcogenetos

Zhang et al. ${ }^{54}$ estudaram sulfetos de Tl, Sn, Pb e Ag e sais sulfurosos de As, Sb e Sn. Determinaram a estequiometria completa ou, ao menos, a proporção entre os constituintes metálicos nas amostras. Por exemplo, foi determinada a estequiometria completa para os compostos $\mathrm{Tl}_{2} \mathrm{~S}, \mathrm{TlS}, \mathrm{Tl}_{4} \mathrm{~S}_{3}$ e $\mathrm{Tl}_{2} \mathrm{~S}_{5}$. A carga gerada na redução dos compostos foi comparada à carga gerada na subsequente oxidação de $\mathrm{Tl}^{0} \mathrm{a} \mathrm{Tl}^{3+}$, o que permitiu uma distinção confiável entre estas fases. A proporção entre $\mathrm{Tl}$ e $\mathrm{Sn}$ nas amostras foi de $\mathrm{Tl}_{4} \mathrm{SnS}_{4}, \mathrm{Tl}_{2} \mathrm{Sn}_{3,5} \mathrm{~S}_{8}$, $\mathrm{Tl}_{2} \mathrm{SnS}_{3}, \mathrm{Tl}_{4} \mathrm{SnS}_{3}, \mathrm{Tl}_{2} \mathrm{Sn}_{2} \mathrm{~S}_{3}$ e Tl $\mathrm{SnS}_{2},{ }^{54}$ enquanto que em outro estudo, a proporção entre $\mathrm{Bi}: \mathrm{Cu}: \mathrm{PB}$ foi de $\mathrm{Bi}_{1,8} \mathrm{~Pb}_{039} \mathrm{Sr}_{1,99} \mathrm{Ca}_{2,06} \mathrm{Cu}_{3,15} \mathrm{O}_{10,5}{ }^{74}$

Outros trabalhos envolvendo o estudo da estequiometria de óxidos metálicos e calcogenetos empregando VIM são mostrados na Tabela 1.

Análise de especiação redox em sólidos

Transformações sólido-para-sólido podem produzir picos voltamétricos que revelam a presença de centros redox estruturalmente diferentes em sólidos inorgânicos. Este fenômeno tem sido demonstrado em numerosos casos de compostos contendo íons metálicos eletroativos em ambientes de coordenação diferentes, como em sítios octaédricos e tetraédricos de óxidos ( $\mathrm{Ti}^{\mathrm{n}+} \mathrm{em}$ amostras de zeólitas) ${ }^{71}$ ou íons $\mathrm{Cu}^{n+}$ em óxidos de diversas composições, exibindo vários comportamentos eletroquímicos..$^{57,58}$ Outros exemplos de especiação redox em amostras sólidas estão listados na Tabela 1 .

\section{Aplicações da VIM na análise e caracterização de compostos orgânicos e organometálicos}

As reações no estado sólido de compostos organometálicos são uma classe muito importante de processos, tendo um papel fundamental em muitos processos naturais (mineralização, corrosão, processos de radiação induzida) e industriais (em cerâmicas, baterias, materiais eletrocrômicos, eletrossínteses, etc.). ${ }^{7}$ Em contraste aos sólidos 
inorgânicos, a maioria dos compostos orgânicos e organometálicos é solúvel em um solvente orgânico apropriado, o que os torna facilmente disponíveis para experimentos eletroquímicos em solução. No entanto, o comportamento eletroquímico destes compostos no estado sólido e em soluções aquosas pode ser bastante diferente ao das espécies dissolvidas, o que faz com que o estudo eletroquímico no estado sólido seja indispensável. A facilidade na preparação do eletrodo e o curto tempo de análise favorecem a VIM nestes estudos.

A exploração da VIM no estudo de compostos orgânicos pode ser feita para diversos propósitos: para abordar questões mecanísticas de reações químicas ou eletroquímicas; para acessar propriedades físicas e químicas de um composto alvo, ou alcançar informação qualitativa ou quantitativa de um composto. Este último, a determinação qualitativa e quantitativa de compostos orgânicos é, atualmente, geralmente um domínio de técnicas cromatográficas e espectrofotométricas. Entretanto, a voltametria e, especialmente, a VIM podem ser altamente úteis como ferramenta para análises rápidas e de baixo custo, sem a necessidade de qualquer etapa de extração da amostra. Por exemplo, Komorsky-Lovric e colaboradores ${ }^{75}$ usaram a VIM na detecção de traços de cocaína em papéis de dinheiro contaminados. Utilizando a voltametria de onda quadrada, determinaram o limite de detecção para a concentração superficial de cocaína como sendo $0,3 \mu \mathrm{g} \mathrm{cm}^{-2}$.

Komorsky-Lovric e Nigovic ${ }^{41}$ utilizaram as técnicas de voltametria cíclica e voltametria de onda quadrada na determinação qualitativa dos fármacos ácido 5-aminosalicílico, ciprofloxacina e azitromicina. Já em outro trabalho, ${ }^{40}$ os mesmos autores investigaram as propriedades eletroquímicas da sinvastatina sólida e em solução, a fim de desenvolver métodos para a análise qualitativa e quantitativa de sinvastatina em formulações farmacêuticas.

Devido às limitações causadas pela imobilização mecânica da amostra à superfície do eletrodo, a informação analítica de um composto alvo é essencialmente de natureza qualitativa. Entretanto, já foi demonstrado que para o rastreamento de pesticidas na superfície de pepino e alface, seguindo-se procedimentos idênticos na preparação do eletrodo, também se conseguem obter informações semiquantitativas. ${ }^{76} \mathrm{O}$ rastreamento rápido fornece informações sobre a presença ou ausência de certos pesticidas e pode, consequentemente, eliminar custos e o tempo gasto em análises desnecessárias como, por exemplo, cromatográficas.

Até agora, maior ênfase ao uso analítico foi posta na aplicação da VIM no estudo de propriedades redox de sólidos orgânicos e nos mecanismos de suas reações eletroquímicas. Estas reações podem proceder através de dissolução, se o produto da reação for solúvel, ou como uma transformação sólido-sólido, se o produto for insolúvel no meio eletrolítico. Existem diversos exemplos de estudos (Tabela 2) mostrando que importantes resultados sobre os processos redox no estado sólido podem ser obtidos com os experimentos voltamétricos.

\section{Aplicações da VIM na Arqueometria}

A VIM tem se tornado um método atrativo para análises de objetos de natureza única e/ou muito valiosos, como obras de arte e materiais arqueológicos. Isto se deve a sua característica de necessitar de uma quantidade extremamente pequena da amostra, sem requerer qualquer forma de pré-tratamento, o que permite que a amostragem seja pouco invasiva ao objeto estudado, além de evitar a remoção do mesmo para o laboratório de ensaio. ${ }^{94,95}$

A identificação de corantes orgânicos e pigmentos empregados em pinturas e tecidos é um fato importante no exame científico de obras de arte e possui um papel essencial nos campos da arqueometria, conservação e restauração de artefatos. ${ }^{94,95} \mathrm{~A}$ identificação dos agentes colorantes presentes nos corantes de pinturas de trabalhos de arte é um tarefa complexa devido à presença simultânea de corantes, pigmentos, outros minerais, polissacarídeos, proteínas, óleos e/ou re-
Tabela 2. Trabalhos envolvendo a análise de compostos orgânicos e organometálicos empregando VIM

\begin{tabular}{|c|c|c|c|}
\hline Analito & Propósito analítico & $\begin{array}{l}\text { Eletrodo de } \\
\text { trabalho }\end{array}$ & Ref. \\
\hline Fulereno $\left(\mathrm{C}_{60}\right)$ & $\begin{array}{c}\text { Estudo de processos de inserção } \\
\text { de íons }\end{array}$ & $\mathrm{Au}, \mathrm{GCE}, \mathrm{Pt}$ & 77,78 \\
\hline $\begin{array}{l}\text { Fito-halocianino } \\
\text { de Co }\end{array}$ & Eletrocatálise em estado sólido & PIGE & 79 \\
\hline $\begin{array}{l}\text { Bisfito-halocianino } \\
\text { de Lu }\end{array}$ & $\begin{array}{c}\text { Estudo de processos de inserção } \\
\text { de íons }\end{array}$ & PIGE & 80 \\
\hline $\begin{array}{l}\text { Complexos } \\
\text { metálicos de } \mathrm{Cr}\end{array}$ & $\begin{array}{c}\text { Detecção de intermediários } \\
\text { instáveis }\end{array}$ & $\mathrm{C}$ pirolítico & 81 \\
\hline Decametilferroceno & $\begin{array}{l}\text { Conversão redox sólido-para- } \\
\text { sólido }\end{array}$ & C pirolítico & 22 \\
\hline Pigmentos & $\begin{array}{l}\text { Comportamento eletroquímico } \\
\text { e quantificação de diversos } \\
\text { pigmentos orgânicos }\end{array}$ & $\begin{array}{l}\text { PIGE, } \\
\text { Compósito }\end{array}$ & $\begin{array}{c}39,82- \\
87\end{array}$ \\
\hline Pesticida & $\begin{array}{c}\text { Detecção qualitativa em pepino } \\
\text { e alface }\end{array}$ & PIGE & 76 \\
\hline Metalocenos & Processos redox em estado sólido & PIGE & 88 \\
\hline $\begin{array}{l}\text { Estruturas orgânicas } \\
\text { metálicas }\end{array}$ & Processos redox em estado sólido & GCE & 89 \\
\hline Complexos de Mn & $\begin{array}{l}\text { Fotoeletroquímica em estado } \\
\text { sólido }\end{array}$ & C pirolítico & 90 \\
\hline $\begin{array}{l}\text { Tetraciano- } \\
\text { quinodimetano }\end{array}$ & $\begin{array}{l}\text { Formação de produtos de } \\
\text { inserção de cátions como } \\
\text { ferramenta analítica }\end{array}$ & $\begin{array}{l}\text { C pirolítico, } \\
\mathrm{Au}\end{array}$ & 91 \\
\hline Fármacos & $\begin{array}{l}\text { Identificação de diferentes } \\
\text { tipos de fármacos em amostras } \\
\text { biológicas e farmacêuticas }\end{array}$ & PIGE & $40,41,92$ \\
\hline Flavonoides & $\begin{array}{l}\text { Identificação de flavonoides em } \\
\text { pigmentos de obras de arte }\end{array}$ & PIGE & 38 \\
\hline Cocaína & Detecção de traços & PIGE & 75 \\
\hline $\begin{array}{l}\text { Hidrocarbonetos } \\
\text { aromáticos }\end{array}$ & $\begin{array}{l}\text { Comportamento eletroquímico } \\
\text { em líquidos iônicos }\end{array}$ & $\mathrm{Au}$ & 93 \\
\hline
\end{tabular}

sinas, os quais complicam a detecção por outros métodos. Entretanto, com a VIM esta identificação se torna possível devido à atividade redox dos cromóforos dos corantes orgânicos e pigmentos presentes nas amostras. Um exemplo é a distinção entre dois pigmentos azuis (azul da Prússia e índigo) usados em camadas coloridas de trabalhos de arte, ilustrado pela Figura 5. ${ }^{82}$

A VIM tem forte potencial para fornecer informações sobre a composição de diferentes tipos de microamostras sólidas, como demonstrado no estudo de azul Maia usado em pinturas de parede de diferentes sítios arqueológicos de Campeche e Yacatán, México. ${ }^{39,83}$ As informações sobre os materiais que constituem uma pintura de parede podem revelar as técnicas aplicadas durante o processo de produção e podem também dar uma indicação da data do trabalho do artefato. Em contato com eletrólitos aquosos ${ }^{39}$ e não aquosos, ${ }^{83}$ as amostras de azul Maia imobilizadas no eletrodo de trabalho (PIGE) forneceram respostas eletroquímicas bem definidas, permitindo a caracterização de diferentes espécies de azul Maia que podem estar correlacionadas com diferentes procedimentos de preparação deste pigmento em diferentes épocas.

\section{CONSIDERAÇÕES FINAIS}

A investigação de micropartículas insolúveis imobilizadas na superfície de um eletrodo tem se tornado um interessante campo da eletroanalítica. A VIM tem ampliado os estudos relacionados ao fenômeno eletroquímico que acontece na interface partícula/solução pelo fato de ser útil para resolver os mais variados problemas como, 


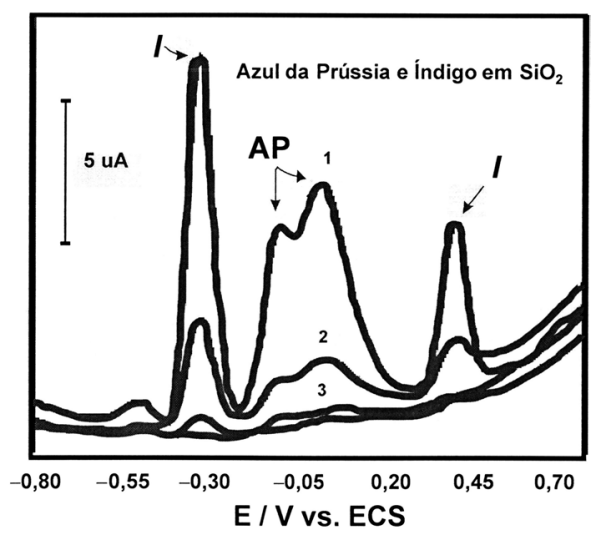

Figura 5. Voltamogramas de onda quadrada de uma mistura em pó de azul da Prússia e índigo diluída com $\mathrm{SiO}_{2}$ (por trituração). Os picos de índigo e azul da Prússia são denotados por I e AP, respectivamente. Concentração dos compostos colorantes: 3,6 e 1,9\% (1); 0,7 e 0,4\% (2); 0,07 e 0,04\% (3); a linha base está demonstrada pela linha pontilhada. Eletrólito suporte: acetato de sódiolácido acético 0,2 $\mathrm{mol} \mathrm{L}^{-1}$; eletrodo de trabalho: PIGE. Adaptada da ref. 82

por exemplo, determinação de dados cinéticos e elucidação das reações eletroquímicas de compostos sólidos, além de possibilitar determinações qualitativas e semiquantitativas da composição de diferentes materiais sólidos

Assim, este método transformou-se em uma importante ferramenta analítica devido as suas facilidades experimentais relacionadas com a preparação do eletrodo, a boa reprodutibilidade e a curta escala de tempo requerida para as medidas eletroanalíticas.

A VIM possibilita a identificação de diversos compostos inorgânicos e orgânicos presentes em amostras sólidas, sem a necessidade de qualquer pré-tratamento ou dissolução preliminar da mesma. O fato de ser um método simples, microdestrutivo e de baixo custo permite a sua ampla aplicabilidade no ramo da ciência arqueológica, indústria e em diferentes campos de pesquisa científica, mostrando-se adequado para os interesses propostos.

\section{AGRADECIMENTOS}

Ao CNPq e à CAPES pelos auxílios e bolsas concedidos; à colaboração do Prof. Dr. F. Scholz (Universidade de Greifswald, Alemanha) pelas discussões e sugestões durante a concepção deste artigo.

\section{REFERÊNCIAS}

1. Kolthoff, I. M.; Laitinen, H. A.; Science 1940, 92, 152.

2. Scholz, F.; Nitschke, L.; Henrion, G.; Naturwissenschaften 1989, 76, 71.

3. Scholz, F.; Nitschke, L.; Henrion, G.; Damaschun, F.; Naturwissenschaften 1989, 76, 167.

4. Fiedler, D. A.; Scholz, F. Em Electroanalytical Methods: Guide to Experiments and Applications; Scholz, F., ed.; Springer: Berlin, 2005, cap. II.8.

5. Scholz, F.; Nitschke, L.; Henrion, G.; Fresen. Z. Anal. Chem. 1989, 334, 56.

6. Scholz, F.; Nitschke, L.; Henrion, G.; Damaschun, F.; Fresen. Z. Anal. Chem. 1989, 335, 189.

7. Grygar, T.; Marken, F.; Schröder, U.; Scholz, F.; Collect. Czech. Chem. Commun. 2002, 67, 163.

8. van Oorschot, I. H. M.; Grygar, T.; Dekkers, M. J.; Earth Planet. Sci. Lett. 2001, 193, 631.

9. Scholz, F.; Lange, B.; Trends Anal. Chem. 1992, 11, 359.

10. Grycar, T.; J. Electroanal. Chem. 1996, 405, 117.
11. Scholz, F.; Meyer, B. Em Electroanalytical Chemistry, A Series of Advances; Bard, A. J.; Rubinstein, I., eds.; Marcel Dekker: New York, 1998, cap. 1.

12. Scholz, F.; Meyer, B.; Chem. Soc. Rev. 1994, 23, 341.

13. Scholz, F.; Schröder, U.; Gulaboski, R.; Electrochemistry of Immobilized Particles and Droplets, Springer: Berlin, 2005.

14. Scholz, F. Em Electrochemical Dictionary; Bard, A. J.; Inzelt, G.; Scholz, F., eds.; Springer: Berlin, 2008, Three-phase boundary.

15. Brainina, Kh.; Neyman, E.; Electroanalytical Stripping Methods, John Wiley and Sons: New York, 1993, cap. 3.

16. Brainina, Kh. Z.; Vydrevich, M. B.; J. Electroanal. Chem. 1981, 121, 1.

17. Doménech-Carbó, A.; Doménech-Carbó, M. T.; Osete-Cortina, L.; Gimeno-Adelantado, J. V.; Bosch-Reig, F.; Mateo-Castro, R.; Talanta 2002, 56, 161 .

18. Doménech-Carbó, A.; Gimeno-Adelantado, J. V.; Bosch-Reig, F.; Sánchez-Ramos, S.; Doménech-Carbó, M. T.; Saurí-Peris, M. C.; Analyst 2001, 126, 1764.

19. Long, J. W.; Ayers, K. E.; Rolison, D. R.; J. Electroanal. Chem. 2002, 522,58 .

20. Jiang, J.; Kucernak, A.; Electrochim. Acta 2000, 45, 2227.

21. Lange, B.; Lovric, M.; Scholz, F.; J. Electroanal. Chem. 1996, 418, 21.

22. Bond, A. M.; Marken, F.; J. Electroanal. Chem. 1994, 372, 125.

23. Bond, A. M.; Colton, R.; Daniels, F.; Fernando, D. R.; Marken, F.; Nagaosa, Y.; van Steveninck, R. F. M.; Walter, J. N.; J. Am. Chem. Soc. 1993, 115, 9556.

24. Kucernak, A. R.; Chowdhury, P. B.; Wilde, C. P.; Kelsall, G. H.; Zhu, Y. Y.; Williams, D. E.; Electrochim. Acta 2000, 45, 4483.

25. Chatterjee, A.; Wiltshire, R.; Holt, K. B.; Compton, R. G.; Foord, J. S.; Marken, F.; Diamond Relat. Mater. 2002, 11, 646.

26. Marken, F.; Compton, R. G.; Goeting, C. H.; Foord, J. S.; Bull, S. D.; Davies, S. G.; J. Solid State Electrochem. 2001, 5, 88.

27. Mancey, D. S.; Shoesmith, D. W.; Lipkowski, J.; McBride, A. C.; Noël, J.; J. Electrochem. Soc. 1993, 140, 637.

28. White, A. F.; Peterson, M. L.; Hochella, M. F.; Geochim. Cosmochim. Acta 1994, 58, 1859.

29. Nicol, M. J.; Needs, C. R. S.; Electrochim. Acta 1975, 20, 585.

30. Nicol, M. J.; Needs, C. R. S.; Electrochim. Acta 1977, 22, 1381.

31. Yin, Q.; Vaughan, D. J.; England, K. E. R.; Kelsall, G. H.; J. Colloid Interface Sci. 1994, 166, 133.

32. Richardson, P. E.; O’Dell, C. S.; J. Electrochem. Soc. 1985, 132, 1350.

33. Wang, X.-H.; Ahlberg, E.; Forssberg, K. S. E.; J. Appl. Electrochem. 1992, 22, 1095

34. Cepriá, G.; Abadías, O.; Pérez-Arantegui, J.; Castillo, J. R.; Electroanalysis 2001, 13, 477.

35. Sedano, A. B. A.; García, M. L. T.; Barbado, M. D. V.; Batanero, P. S.; J. Electroanal. Chem. 2004, 566, 433.

36. Cepriá, G.; Usón, A.; Pérez-Arantegui, J.; Castillo, J. R.; Anal. Chim. Acta 2003, 477, 157.

37. Komorsky-Lovric, S.; Mirceski, V.; Scholz, F.; Mikrochim. Acta 1999, $132,67$.

38. Doménech-Carbó, A.; Doménech-Carbó, M. T.; Saurí-Teris, M. C.; Talanta 2005, 66, 769.

39. Doménech, A.; Doménech-Carbó, M. T.; Pascual, M. L. V. A.; Anal. Chem. 2007, 79, 2812.

40. Komorsky-Lovric, S.; Nigovic, B.; J. Electroanal. Chem. 2006, 593, 125.

41. Komorsky-Lovric, S.; Nigovic, B.; J. Pharm. Biomed. Anal. 2004, 36, 81.

42. Komorsky-Lovric, S.; Vukasinovic, N.; Penovski, R.; Electroanalysis 2003, 15, 544 .

43. Hermes, M.; Scholz, F.; Electrochem. Commun. 2000, 2, 845.

44. Gruner, W.; Kunath, J.; Kalnishevskaja, L. N.; Posokin, J. V.; Brainina, Kh. Z.; Electroanalysis 1993, 5, 243. 
45. Scholz, F.; Müller, W.-D.; Nitschke, L.; Rabi, F.; Livanova, L.; Fleischfresser, C.; Thierfelder, Ch.; Fresenius' J. Anal. Chem. 1990, 338, 37.

46. Scholz, F.; Rabi, F.; Müller, W.-D.; Electroanalysis 1992, 4, 339.

47. Lux, L.; Gálová, M.; Hezelová, M.; Markusová, K.; J. Solid State Electrochem. 1999, 3, 288.

48. Gálová, M.; Orináková, R.; Grygar, T.; Lux, L.; Hezelová, M.; Part. Sci. Technol. 2001, 19, 85

49. Scholz, F.; Nitschke, L.; Henrion, G.; Electroanalysis 1990, $2,85$.

50. Doménech-Carbó, A.; Doménech-Carbó, M. T.; Osete-Cortina, L.; Gimeno-Adelantado, J. V.; Sánchez-Ramos, S.; Bosch-Reig, F.; Electroanalysis 2003, 15, 1465.

51. Dómenech-Carbó, A.; Dómenech-Carbó, M. T.; Moyá-Moreno, M.; Gimeno-Adelantado, J. V.; Bosch-Reig, F.; Anal. Chim. Acta 2000, 407, 275.

52. Carbonelle, P.; Lamberts, L.; Electrochim. Acta 1992, 37, 1321.

53. Meyer, B.; Zhang, S.; Scholz, F.; Fresenius' J. Anal. Chem. 1996, 356, 267.

54. Zhang, S.; Meyer, B.; Moh, G. H.; Scholz, F.; Electroanalysis 1995, 7, 319.

55. Bond, A. M.; Scholz, F.; J. Geochem. Explor. 1992, 42, 227.

56. Cepriá, G.; García-Gareta, E.; Pérez-Arantegui, J.; Electroanalysis 2005 , 17, 1078 .

57. Scholz, F.; Nitschke, L.; Kemnitz, E.; Olesch, T.; Henrion, G.; Hass, D.; Bagchi, R. N.; Herrmann, R.; Pruss, N.; Wilde, W.; Fresenius' J. Anal. Chem. 1989, 335, 571.

58. Scheurell, S.; Scholz, F.; Olesch, T., Kemnitz, E.; Supercond. Sci. Technol. 1992, 5, 303.

59. Sedano, A. B. A.; García, M. L. T.; Barbado, M. D. V.; Batanero, P. S.; J. Solid State Electrochem. 2003, 7, 301.

60. Doménech-Carbó, M. T.; Casas-Catalán, M. J.; Doménech-Carbó, A.; Mateo-Castro, R.; Gimeno-Adelantado, J. V .; Bosch-Reig, F.; Fresenius' J. Anal. Chem. 2001, 369, 571.

61. Doménech-Carbó, A.; Doménech-Carbó, M. T.; Gimeno-Adelantado, J. V.; Bosch-Reig, F.; Saurí-Peris, M. C.; Casas-Catalán, M. J.; Fresenius' J. Anal. Chem. 2001, 369, 576.

62. Grygar, T.; J. Solid State Electrochem. 1997, 1, 77.

63. Grygar, T.; van Oorschot, I. H. M.; Electroanalysis 2002, 14, 339.

64. Doménech-Carbó, A.; Sánchez-Ramos, S.; Doménech-Carbó, M. T.; Gimeno-Adelantado, J. V.; Bosch-Reig, F.; Yusá-Marco, D. J.; SauríPeris, M. C.; Electroanalysis 2002, 14, 685.

65. Komorsky-Lovric, S.; Croat. Chem. Acta 1998, 71, 263.

66. Fiedler, D. A.; Basenhard, J. O.; Fooken, M. H.; J. Power Sources 1997, 69, 157.

67. Doménech-Carbó, A.; Doménech-Carbó, M. T.; Osete-Cortina, L.; Electroanalysis 2001, 13, 927.

68. Centeno, B.; Tascón, M. L.; Vásquez, M. D.; Sánchez Batanero, P.; Electrochim. Acta 1991, 36, 277.
69. Doménech-Carbó, A.; Doménech-Carbó, M. T.; Gimeno-Adelantado, J. V.; Moya-Moreno, M.; Bosch-Reig, F.; Electroanalysis 2000, 12, 120.

70. Adekola, F. A.; Colin, C.; Bauer, D.; Electrochim. Acta 1992, 37, 2009.

71. de Castro-Martins, S.; Khouzami, S.; Tuel, A.; Bem Taarit, Y.; El Murr, N.; Sellami, A.; J. Electroanal. Chem. 1993, 350, 15.

72. Doménech, A.; Corma, A.; García, H.; Valencia, S.; Top. Catal. 2000, 11,401 .

73. Bodoardo, S.; Geobaldo, F.; Penazzi, N.; Arrabito, M.; Rivetti, F.; Spano, G.; Lamberti, C.; Zecchina, A.; Electrochem. Commun. 2000, $2,349$.

74. Scholz, F.; Lange, B.; Fresenius' J. Anal. Chem. 1990, 338, 293.

75. Komorsky-Lovric, S.; Galic, I.; Penovski, R.; Electroanalysis 1999, 11, 120.

76. Reddy, S. J.; Hermes, M.; Scholz, F.; Electroanalysis 1996, 8, 955.

77. Chlistunoff, J.; Cliffel, D.; Bard, A. J.; Thin Solid Films 1995, 257, 166.

78. Bond, A. M.; Miao, W.; Raston, C. L.; J. Phys. Chem. B 2000, 104, 2320.

79. Komorsky-Lovric, S.; Lovric, M.; Scholz, F.; Mikrochim. Acta 1997, 127, 95.

80. Komorsky-Lovric, S.; Quentel, F.; L'Her, M.; Scholz, F.; J. Solid State Electrochem. 2008, 12, 165.

81. Bond, A. M.; Colton, R.; Marken, F.; Walter, J. N.; Organometallics 1994, 13, 5122 .

82. Grygar, T.; Kucková, S.; Hradil, D.; Hradilová, D.; J. Solid State Electrochem. 2003, 7, 706.

83. Doménech, A.; Doménech-Carbó, M. T.; Pascual, M. L. V. A.; J. Phys. Chem. C 2007, 111, 4585.

84. Bosch-Reig, F.; Doménech-Carbó, A.; Doménech-Carbó, M. T.; Gimeno-Adelantado, J. V.; Electroanalysis 2007, 19, 1575.

85. Doménech-Carbó, A.; Doménech-Carbó, T.; Saurí-Peris, C.; GimenoAdelantado, J. V.; Bosch-Reig, F.; Microchim. Acta 2005, 152, 75.

86. Doménech, A.; Doménech-Carbó, M. T.; Pascual, M. L. V. A.; J. Phys. Chem. B 2006, 110, 6027.

87. Doménech, A.; Doménech-Carbó, M. T.; del Río, M. S.; Pascual, M. L. V. A.; J. Solid State Electrochem. 2009, 13, 869.

88. Bond, A. M.; Scholz, F.; Langmuir 1991, 7, 3197.

89. Doménech, A.; García, H.; Doménech-Carbó, M. T.; Llabrés-i-Xamena, F.; J. Phys. Chem. C 2007, 111, 13701.

90. Eklund, J. C.; Bond, A. M.; J. Am. Chem. Soc. 1999, 121, 8306.

91. Bond, A. M.; Fletcher, S.; Marken, F.; Shaw, S. J.; Symons, P. G.; J. Chem. Soc., Faraday Trans. 1996, 92, 3925.

92. Nigovic, B.; Komorsky-Lovric, S.; Devcic, D.; Croat. Chem. Acta 2008 , $81,453$.

93. Chen, A.; Rogers, E. I.; Compton, R. G.; Electroanalysis 2009, 21, 29.

94. Doménech-Carbó, A.; J. Solid State Electrochem. 2010, 14, 363.

95. Doménech-Carbó, A.; Doménech-Carbó, M. T.; Costa, V. Em Electrochemical Methods in Archaeometry, Conservation and Restoration; Scholz, F., ed.; Springer: Berlin, 2009, cap. 2. 